\title{
CINÉTICA DE BIOACUMULACIÓN DE HIDROCARBUROS AROMÁTICOS POLICÍCLICOS EN Anadara similis (ADAMS, 1852) Y Anadara tuberculosa (SOWERBY, 1833) (ARCOIDA: ARCIDAE)
}

\section{KINETICS OF BIOACCUMULATION OF POLYCYCLIC AROMATIC HYDROCARBONS IN Anadara similis (ADAMS, 1852) AND Anadara tuberculosa (SOWERBY, 1833) (ARCOIDA: ARCIDAE)}

\author{
Mónica Zambrano $^{1^{*}}$, Robinson Casanova ${ }^{2^{*}}$, Gustavo Arencibia ${ }^{3}$, Alexis Vidal ${ }^{4}$, Norberto Capetillo ${ }^{5}$
}

\begin{abstract}
${ }^{1}$ Estudiante de Doctorado en Ciencias Biológicas. Centro de Investigaciones Marinas CIM. Universidad de la Habana. La Habana. Cuba. monyzambrano@yahoo.com; ²Magister Gestión e Ingeniería Ambiental. Jefe Laboratorio de Química. *Grupo de Protección del Medio Marino. Centro de Investigaciones Oceanográficas e Hidrográficas del Pacífico Cccp, Tumaco Colombia. rcasanova@dimar.mil.co; ${ }^{3}$ Doctor en Ciencias en el Uso, Manejo y Preservación de los Recursos. Centro de Investigaciones Pesqueras CIP. La Habana, Cuba. garen04@gmail.com; ${ }^{4}$ Doctor en Ciencias Farmacéuticas. Profesor Titular. Grupo de Farmacología-Toxicología, Facultad de Biología, Universidad de La Habana. La Habana, Cuba. alexis.vidal@ infomed.sld.cu; ${ }^{5}$ Magister en Biología Marina y Acuicultura. Estudiante Doctorado Ciencias Marinas, Depto de Pesquería y Biología Marina, La Paz, Baja California Sur, México, norbertcap@yahoo.com
\end{abstract}

Rev. U.D.C.A Act. \& Div. Cient. 15(2): 291 - 302, 2012

\section{RESUMEN}

Se determinó la bioacumulación de hidrocarburos aromáticos policíclicos (HAP) en las los moluscos bivalvos Anadara similis y $A$. tuberculosa, siendo esta última especie empleada como organismo biomonitor, en el Pacífico colombiano. Un total de 120 organismos de cada especie fueron expuestos a una mezcla de los 16 HAP considerados contaminantes prioritarios, por la Environmental Protection Agency (US EPA), suministrados a concentraciones establecidas a partir de niveles considerados seguros para la vida acuática. Los organismos fueron expuestos a una concentración de $15,48 \mathrm{ng} / \mathrm{mL}$ de la mezcla de HAP, por un periodo de 45 días, para A. tuberculosa y 30, para A. similis; la prueba con esta última especie tuvo menor duración, debido a la mortalidad registrada durante el ensayo, la cual, se asocia con la duración del periodo de aclimatación, que abarcó 10 días. Los máximos niveles de acumulación registrados en A. tuberculosa $\left(48,00 \times 10^{2} \mathrm{ng} / \mathrm{g}\right)$ y A. similis $\left(75,00 \times 10^{2}\right.$ $\mathrm{ng} / \mathrm{g}$ ), se presentaron luego de 30 y 25 días de exposición, respectivamente, evidenciándose una mayor capacidad de bioacumulación por parte de A. similis y, por ende, un alto potencial de la misma, como organismo biomonitor de las condiciones ambientales.
Palabras clave: Bioacumulación, Hidrocarburos Aromáticos Policíclicos (HAP), Bivalvos.

\section{SUMMARY}

The bioaccumulation of aromatic polycyclic hydrocarbons (PAH`s) in the bivalve mollusk Anadara similis and A. tuberculosa, was determined, being the latter species used as bio-monitor organism in the Colombian Pacific. A total of 120 organisms of each species were exposed to a mixture of $16 \mathrm{APH}$ considered priority pollutants to the Environmental Protection Agency (US EPA), supplied to established concentrations from levels considered safety to the aquatic life. The organisms were exposed to concentrations of $15.48 \mathrm{ng} / \mathrm{mL}$ of PAH's mixture for a period of 45 days to $A$. tuberculosa and 30 days to $A$. similis, the test with the latter species had less duration due to the mortality registered during the test, associated with the acclimatization period that included 10 days. The highest accumulation levels registered in A. tuberculosa $\left(48.00 \times 10^{2} \mathrm{ng} / \mathrm{g}\right)$ and A. similis $\left(75.00 \times 10^{2} \mathrm{ng} / \mathrm{g}\right)$ were detected, respectively, after 30 and 25 days of exhibition, demonstrating a major bioaccumulation 
capacity of A. similis and for therefore a high potential of this organism as bio-monitor of the environmental conditions.

Key words: Bioaccumulation, Aromatic Polycyclic Hydrocarbons (HAP), Bivalves.

\section{INTRODUCCIÓN}

Los hidrocarburos aromáticos policíclicos (HAP) son constituyentes naturales del petróleo crudo y conforman una fracción de hasta el $20 \%$ del total de hidrocarburos, siendo, a la vez, el grupo potencialmente más tóxico (Eisler, 2000). Se conocen aproximadamente $100 \mathrm{HAP}$ en el aire, en el suelo, en los alimentos y en el agua (Zedeck, 1980); sin embargo, solo 16 son considerados de interés en función de sus aplicaciones industriales y como contaminantes ambientales tóxicos, por la EPA (Achten \& Hofmann, 2009; Rey-Salgueiro et al. 2009), la ATSDR (2011) y la IARC (1983), fundamentalmente, por sus efectos mutagénicos y cancerígenos (Potin et al. 2004). Estos compuestos, se encuentran entre los más comunes contaminantes orgánicos persistentes en ambientes acuáticos (Nesto et al. 2010), que causan efectos biológicos a largo plazo y su toxicidad sobre organismos acuáticos, se incrementa con la exposición a la radiación solar y por la por fotoactivación de las moléculas (Pelletier et al. 2006; Bellas et al. 2008; Okay \& Karacik, 2008).

Para realizar estudios de seguimiento a este tipo de contaminantes en ambientes acuáticos se ha recurrido al uso de bivalvos, como organismos biomonitores (Page et al. 2005; Chęć et al. 2008), considerando al mejillón entre los mejores centinelas en el seguimiento de la contaminación marina, por ser organismos sedentarios, filtradores y por poseer una limitada capacidad de metabolización (SorianoSanz et al. 2006); sin embargo, en el Pacífico colombiano su presencia es escasa, situación que generó la necesidad de seleccionar una especie representativa de la zona.

En Colombia, las especies de moluscos bivalvos Anadara similis y A. tuberculosa, conocidas comúnmente como Piangua, son ampliamente explotadas y cuentan con una alta demanda en el mercado, por ende, son de gran interés comercial, llegando a constituir el $96 \%$ de la producción total de bivalvos del país (INCODER, 2007). Ambas especies habitan en el ecosistema de manglar, en especial, entre las raíces de mangle rojo (Rhizophora mangle), siendo A. tuberculosa más abundante, razón por la que sobre la misma, se realiza un importante esfuerzo de captura, que la ha llevado a ser catalogada como especie vulnerable (Ardila et al. 2002). Si bien A. similis no se encuentra catalogada dentro de este grupo es extraída de manera simultánea durante las faenas de recolección y teniendo en cuenta su menor abundancia es muy factible que se encuentre bajo las mismas condiciones de vulnerabilidad.

Debido a la importancia comercial con que cuentan ambas especies y, en particular A. tuberculosa, su amplia distribución en el Pacífico colombiano y su condición de organismo sésil y filtrador, ha sido utilizada para predecir exposición, efectos y susceptibilidad ante la presencia de sustancias antropogénicas (Sericano et al. 1995); en Colombia (Marrugo, 1993; Casanova, 1995; Calero \& Zambrano, 1997; Velásquez López \& Cortes, 1997) y en diversos países de la región (De la Cruz, 1994; Lauenstein, 1995; Sericano et al. 1995; Beliaeff et al. 1997; Herrero et al. 1999).

Teniendo en cuenta lo anterior, en el presente estudio, se evaluó la capacidad de bioacumulación de ambas especies al ser expuestas a una mezcla de 16 HAP, comparando las respuestas presentadas entre ellas, bajo las mismas condiciones experimentales. Así mismo, se realizó un seguimiento al comportamiento de incorporación y de depuración de los compuestos, lo que permitirá, adicionalmente, determinar periodos de cuarentena, en caso de eventos de contaminación asociados a hidrocarburos, tendientes a proteger la salud de los consumidores.

\section{MATERIALES Y MÉTODOS}

Los organismos de ensayo, se recolectaron en la Bahía de Tumaco, en el Pacífico colombiano, cuyas coordenadas corresponden a los $1^{\circ} 53^{\prime} 10^{\prime \prime} \mathrm{N}$ y $78^{\circ} 39^{\prime} 50^{\prime \prime}$ W (Figura 1) y fueron adquiridos a través de pescadores artesanales, trasladados al laboratorio del Centro de Investigaciones Oceanográficas e Hidrográficas del Pacífico - CCCP, en donde fueron sometidos a un periodo de aclimatación en tanques de 250L.

La duración de este periodo abarcó 10 días, con el fin que los organismos no solamente se adaptaran a las condiciones de laboratorio, sino que también depuraran los niveles de compuestos de alto peso molecular (PM), que pudieran haber acumulado en el medio antes de la captura, pues estos compuestos tienen una mayor persistencia con respecto a los de bajo y medio PM. Este periodo, se determinó teniendo como referencia el hecho que los bivalvos marinos utilizados en pruebas similares requieren de dos días de depuración (APHA, AWWA, WEF, 2005).

El agua marina usada para el desarrollo de los experimentos fue tomada de un área aledaña a las instalaciones del CCCP (Figura 1), durante los periodos de marea alta, empleando una motobomba que la impulsaba hacia unos tanques de almacenamiento, pasando, posteriormente, a través de un filtro para su uso en las pruebas. 


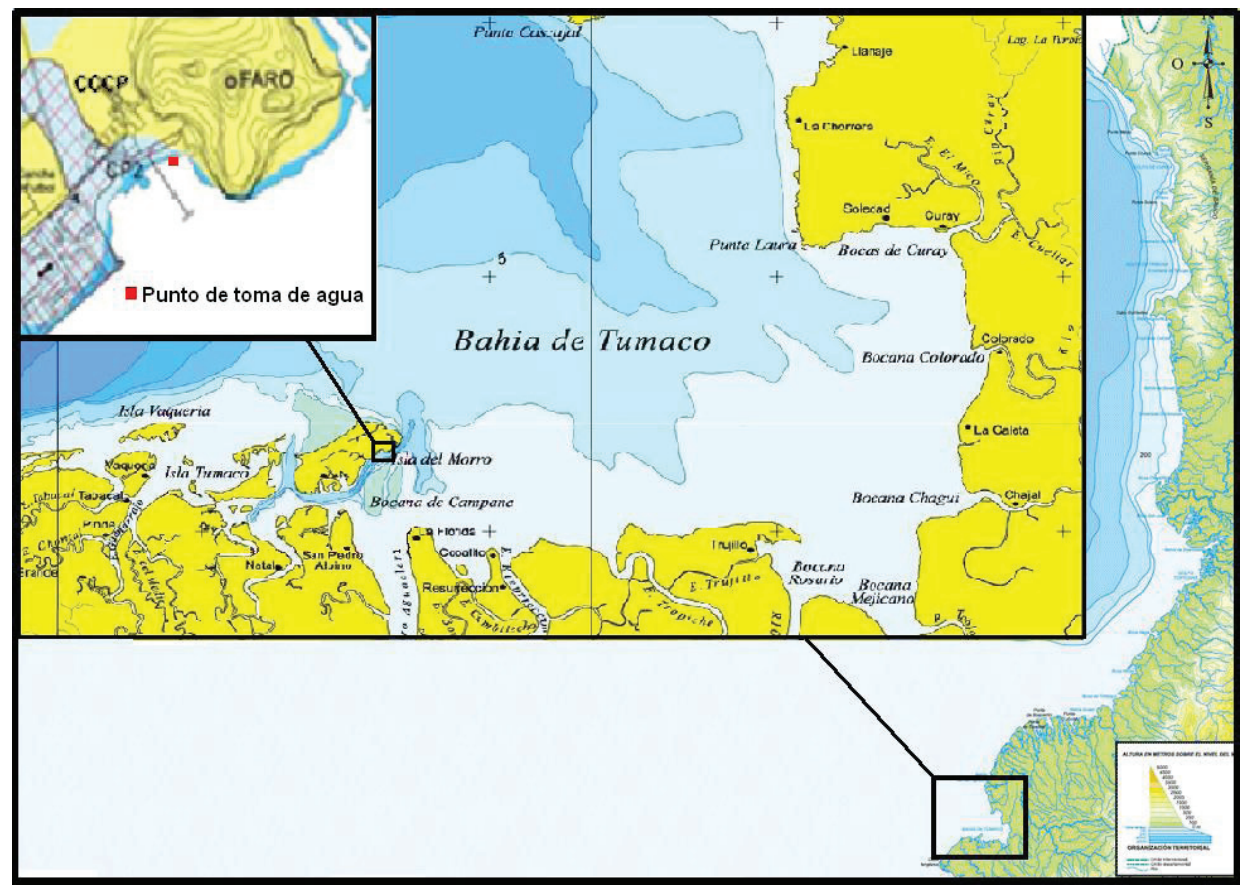

Figura 1. Bahía de Tumaco.

Las pruebas realizadas fueron de tipo semiestático, con recambios del $100 \%$ del volumen de agua (20L), cada 24 horas, en todos los recipientes: los dos tipos de control (agua marina filtrada y con adición de acetona) y, en los recipientes de ensayo, con la solución. No se realizó suministro de aireación durante las pruebas, por lo que el agua de recambio se sometía previamente a varias horas de aireación, con el fin de saturar de oxígeno disuelto (OD).

Los controles empleados consistían en mantener un grupo de organismos en los recipientes de ensayo en agua marina, pero sin adición de la solución (mezcla de HAP), por lo que se tenía un control con solo agua de disolución (agua marina filtrada) y otro al que adicionalmente se añadía el solvente (acetona), con un volumen igual al empleado en la adición del compuesto (15mL); en ambos casos, se trabajaron por triplicado.

Como recipientes de ensayo, se utilizaron acuarios de vidrio aforados a 20,0L y, en cada uno de ellos, se expusieron un total de 10 organismos. La prueba consistió en exponer 120 organismos de cada especie (A. similis y A. tuberculosa) a una mezcla de los $16 \mathrm{HAP}$, alcanzando una concentración de $15,48 \mathrm{ng} / \mathrm{mL}$ en los recipientes de ensayo. Culminado el periodo de aclimatación, se tomó una muestra de 10 organismos, para determinar los niveles de los compuestos a evaluar que pudieran presentar los organismos.

Las concentraciones establecidas para cada compuesto en la mezcla equivalen a los niveles guía de calidad del
Canadian Council of Ministres of the Environment (CCME, 2010), los cuales, no representan riesgo para la vida acuática (estos niveles solamente se encuentran estipulados para ecosistemas dulceacuícolas). Para los compuestos que no cuentan con un nivel de referencia, se estableció una concentración inferior a la estipulada para el antraceno, que cuenta con la mayor restricción entre los compuestos que pertenecen a este grupo dentro de la guía.

La mezcla de HAP requirió la preparación de tres soluciones patrón (de acuerdo a la similitud en la concentración requerida para cada compuesto), empleando acetona como solvente, que fueron adicionadas en diferentes alícuotas, con el fin de obtener las concentraciones estipuladas para adelantar la prueba, obteniendo una concentración final de 15,48 ng/mL (de los 16 analitos), requerida en los recipientes de ensayo. La composición de la mezcla al igual que las características fisicoquímicas de cada compuesto, se presenta en la tabla 1.

Diariamente, se registraron parámetros fisicoquímicos, tales como: salinidad, $\mathrm{pH}$, temperatura y $\mathrm{OD}$, empleando un multiparámetros WTW Multi 340i. Así mismo, se proporcionó alimento a los organismos, suministrando 1,0L de diatomeas (con una densidad promedio de $3 \times 10^{6}$ células/ $\mathrm{mL}$ ) en cada recipiente de ensayo, tras realizar el recambio. Las diatomeas, se cultivaron en el laboratorio, a partir de células obtenidas tras filtrar agua marina en la zona de la que se abastecía el laboratorio. 


\begin{tabular}{|c|c|c|c|c|c|c|c|c|c|c|c|c|c|c|c|c|c|}
\hline 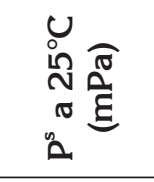 & $\begin{array}{l}\circ \\
\stackrel{\circ}{=} \\
=\end{array}$ & $\begin{array}{l}\text { \& } \\
0 \\
0\end{array}$ & ठั & $\begin{array}{l}\hat{\sigma} \\
\tilde{\sigma}\end{array}$ & $\hat{\circ}$ & $\stackrel{\llcorner}{\sim}$ & $\begin{array}{l}\stackrel{\infty}{N} \\
\stackrel{m}{\sim}\end{array}$ & $\mid \begin{array}{l}0 \\
0 \\
x \\
m \\
m \\
\sigma \\
\sigma\end{array}$ & 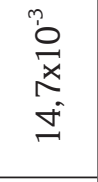 & & $\begin{array}{l}0 \\
0 \\
x \\
0 \\
i\end{array}$ & $\begin{array}{l}0 \\
0 \\
x \\
\hat{x} \\
m \\
0\end{array}$ & 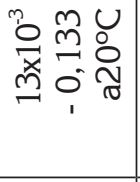 & $\begin{array}{l}\stackrel{n}{0} \\
\stackrel{x}{x} \\
\end{array}$ & & & $\ddot{\ddot{D}}$ \\
\hline 山 $\underset{\sim}{\stackrel{0}{\stackrel{\nu}{N}}}$ & $\begin{array}{l}0 \\
\text { M } \\
\stackrel{N}{ } \\
\text { O }\end{array}$ & & $\begin{array}{l}0 \\
\infty \\
o \\
0 \\
0\end{array}$ & 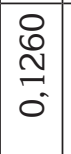 & 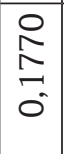 & 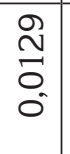 & 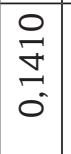 & \begin{tabular}{|l|}
0 \\
0 \\
0 \\
0 \\
0
\end{tabular} & $\begin{array}{l}\text { No } \\
\text { Oे } \\
0\end{array}$ & $\begin{array}{l}\Re \\
\stackrel{2}{0} \\
8 \\
0\end{array}$ & $\begin{array}{l}\stackrel{0}{N} \\
\vdots \\
0 \\
0\end{array}$ & $\begin{array}{l}\stackrel{0}{N} \\
\text { ⿵े } \\
0 \\
0\end{array}$ & $\begin{array}{l}\stackrel{1}{\infty} \\
\tilde{m}_{0} \\
0 \\
0 .\end{array}$ & & $\begin{array}{l}\tilde{N} \\
\text { ஜे } \\
0 \\
0\end{array}$ & $\begin{array}{l}\text { 웅 } \\
8 \\
0\end{array}$ & $\begin{array}{l}\stackrel{0}{\sigma} \\
\frac{0}{2} \\
\frac{0}{0}\end{array}$ \\
\hline$\sum_{\substack{m \\
\tilde{U}}}^{\bar{g}}$ & $\stackrel{\infty}{\underset{J}{J}}$ & & $\stackrel{m}{\simeq}$ & $\begin{array}{l}\infty \\
\infty \\
-\end{array}$ & த & مِ & $\vec{\sim}$ & $\underset{\sim}{\stackrel{\nabla}{v}}$ & $\stackrel{\infty}{\stackrel{\sim}{\sim}}$ & I & $\begin{array}{l}\infty \\
\stackrel{\leftrightarrow}{\sim}\end{array}$ & $\stackrel{\mathscr{\omega}}{\sim}$ & $\begin{array}{l}\infty \\
\stackrel{\sim}{\sigma}\end{array}$ & & $\stackrel{\hat{N}}{\sim}$ & ৪ & 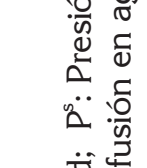 \\
\hline 㟔。 & $\stackrel{\infty}{\sim}$ & $\stackrel{?}{\stackrel{D}{\sim}}$ & $\stackrel{N}{N}$ & ని & $\stackrel{\text { mे }}{m}$ & ㅇํㄹ & 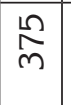 & $\begin{array}{l}8 \\
0 \\
\text { m }\end{array}$ & $\stackrel{\stackrel{n}{m}}{\mathscr{\gamma}}$ & 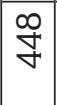 & $\underset{q}{\infty}$ & 号 & 免 & & $\stackrel{\llcorner}{\sim}$ & 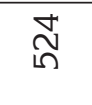 & ס \\
\hline ص & \begin{tabular}{l}
0 \\
0 \\
\multirow{x}{x}{} \\
\\
\end{tabular} & \begin{tabular}{l} 
io \\
\multirow{x}{x}{} \\
$\stackrel{n}{n}$
\end{tabular} & $\begin{array}{l}0 \\
0 \\
x \\
6 \\
i \\
\end{array}$ & 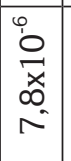 & 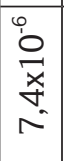 & \begin{tabular}{|l|}
0 \\
0 \\
\\
\\
\end{tabular} & $\begin{array}{l}0 \\
0 \\
0 \\
x \\
m \\
0 \\
0\end{array}$ & 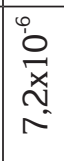 & $\begin{array}{l}0 \\
\dot{0} \\
x \\
\sigma\end{array}$ & $\mid$\begin{tabular}{|c|}
0 \\
0 \\
$x$ \\
\\
0 \\
0
\end{tabular} & 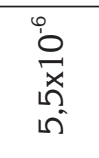 & $\begin{array}{l}0 \\
0 \\
\bar{x} \\
0 \\
0\end{array}$ & 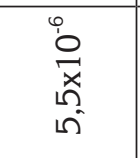 & $\begin{array}{l}0 \\
1 \\
0 \\
\vec{x} \\
\dot{v} \\
\dot{\sigma}\end{array}$ & 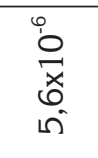 & 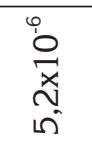 & 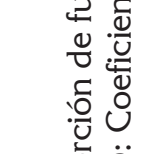 \\
\hline$I$ & $\begin{array}{l}\tilde{\sigma} \\
\stackrel{\gamma}{\gamma}\end{array}$ & & בิ & $\begin{array}{l}\hat{\infty} \\
\stackrel{N}{N}\end{array}$ & 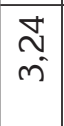 & $\begin{array}{l}\mathscr{Q} \\
\text { m. }\end{array}$ & $\underset{0}{\tilde{O}}$ & $\begin{array}{l}\sigma \\
\sigma \\
\sigma\end{array}$ & 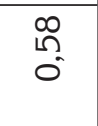 & $\begin{array}{l}0 \\
\infty \\
10\end{array}$ & $\begin{array}{l}0 \\
0 \\
0 \\
0\end{array}$ & \begin{tabular}{l}
0 \\
\multirow{0}{0}{} \\
0
\end{tabular} & & & 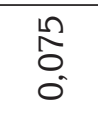 & & ஜ் \\
\hline 资 & $\stackrel{n}{m}$ & $\begin{array}{l}0 \\
\dot{\nabla}\end{array}$ & $\stackrel{m}{m}$ & $\begin{array}{l}\infty \\
\stackrel{\sim}{\sim} \\
\sim\end{array}$ & $\stackrel{\sim}{\sigma}$ & 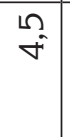 & $\overrightarrow{0}$ & $\stackrel{9}{\sigma}$ & $\begin{array}{l}0 \\
\dot{n}\end{array}$ & $\begin{array}{l}\tilde{G} \\
-i\end{array}$ & $\begin{array}{l}\mathscr{0} \\
0\end{array}$ & $\begin{array}{l}\circ \\
0\end{array}$ & $\begin{array}{l}8 \\
0 \\
0\end{array}$ & $\begin{array}{l}\text { ㅇ } \\
6\end{array}$ & $\begin{array}{l}\text { 오 } \\
0\end{array}$ & 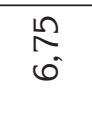 & 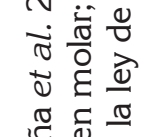 \\
\hline 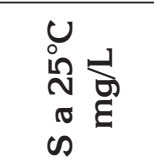 & $\vec{m}$ & $\begin{array}{l}m \\
\text { m. } \\
\text { - }\end{array}$ & $\begin{array}{l}m \\
\text { ñ } \\
m\end{array}$ & 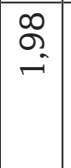 & $\stackrel{\text { D }}{\rightarrow}$ & $\begin{array}{l}\text { 年 } \\
0 \\
0 \\
0\end{array}$ & 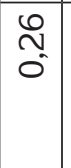 & $\begin{array}{l}\stackrel{n}{m} \\
\vdots \\
0\end{array}$ & $\begin{array}{l}\hat{2} \\
8 \\
0 \\
0\end{array}$ & $\mid \begin{array}{l}\infty \\
8 \\
8 \\
0 \\
0\end{array}$ & 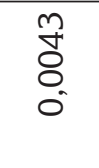 & $\begin{array}{l}\infty \\
\\
8 \\
0\end{array}$ & $\begin{array}{l}\stackrel{\nabla}{0} \\
0 \\
0\end{array}$ & 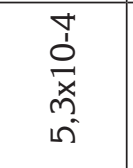 & 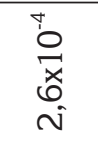 & $\begin{array}{l}8 \\
8 \\
8 \\
0\end{array}$ & 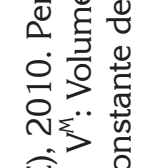 \\
\hline 㞤。 & $\begin{array}{l}n \\
\infty \\
\infty\end{array}$ & ถุ & คุ & $\begin{array}{l}\tilde{L}^{\prime} \\
\mathscr{0}^{\prime} \\
=\end{array}$ & 흠 & $\begin{array}{l}0 \\
\stackrel{\sim}{N}\end{array}$ & $\Xi$ & $\stackrel{\circ}{\llcorner}$ & $\underset{\sigma}{\widetilde{\sigma}}$ & $\stackrel{\llcorner}{\sim}$ & $\stackrel{\sim}{\sim}$ & I & $\underset{0}{0}$ & ఠ & $\stackrel{N}{\sim}$ & $\stackrel{?}{\stackrel{\sim}{N}}$ & 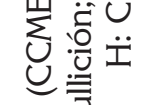 \\
\hline$\sum_{\Sigma} \frac{\bar{\sigma}}{\tilde{\sigma}}$ & $\begin{array}{l}\stackrel{0}{-} \\
\stackrel{0}{0} \\
\stackrel{\sim}{\sim}\end{array}$ & 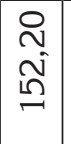 & $\begin{array}{l}\vec{\sim} \\
\stackrel{\Delta}{n} \\
\stackrel{n}{\sim}\end{array}$ & $\begin{array}{l}8 \\
0 \\
0\end{array}$ & 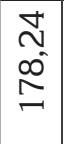 & 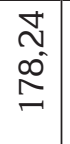 & 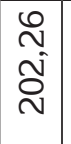 & 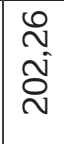 & $\begin{array}{l}8 \\
\text { ô } \\
\text { N }\end{array}$ & 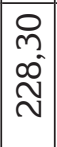 & $\begin{array}{l}\text { N } \\
\text { Nิ } \\
\text { Nิ }\end{array}$ & $\begin{array}{l}\tilde{N} \\
\text { Ñ } \\
\text { N̂}\end{array}$ & $\begin{array}{l}\text { Nิ } \\
\text { Nิ } \\
\text { Nิ }\end{array}$ & $\begin{array}{l}8 \\
\stackrel{6}{0} \\
\stackrel{0}{0}\end{array}$ & $\begin{array}{l}\stackrel{+}{m} \\
\underbrace{\circ} \\
\stackrel{N}{v}\end{array}$ & $\begin{array}{l}0 \\
m \\
\infty \\
\stackrel{0}{N}\end{array}$ & 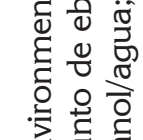 \\
\hline 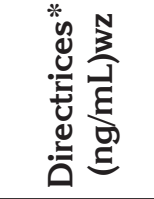 & 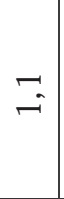 & & $\begin{array}{l}\infty \\
\tilde{n}^{-}\end{array}$ & $\begin{array}{l}\circ \\
M^{-}\end{array}$ & $\stackrel{+}{\sigma_{0}}$ & \begin{tabular}{l}
\multirow{1}{*}{} \\
$\vdots$ \\
0
\end{tabular} & $\begin{array}{l} \\
0 \\
0\end{array}$ & 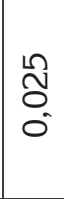 & 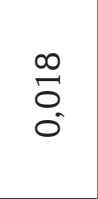 & & & $\begin{array}{l}\stackrel{1}{0} \\
\vdots \\
0 \\
0\end{array}$ & & & & & 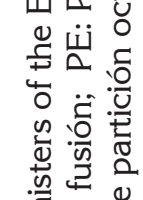 \\
\hline 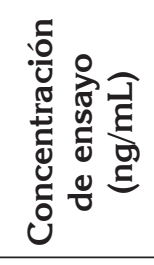 & 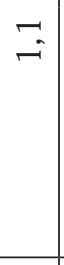 & $\begin{array}{l}0 \\
\text { in }\end{array}$ & $\begin{array}{l}\infty \\
\sim^{\prime}\end{array}$ & $\begin{array}{l}\circ \\
m\end{array}$ & $\sigma_{0}^{+}$ & $\begin{array}{l}\tilde{N} \\
\stackrel{0}{1} \\
x \\
\sim \\
\end{array}$ & $\mid \begin{array}{c}\overrightarrow{0} \\
0 \\
x \\
x \\
\dot{t} \\
0\end{array}$ & $\begin{array}{l}0 \\
0 \\
x \\
n \\
1 \\
N\end{array}$ & $\begin{array}{l}\text { No } \\
\underset{x}{x} \\
\infty \\
-i\end{array}$ & 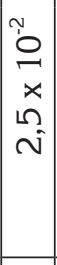 & $\begin{array}{l}\overrightarrow{0} \\
x \\
x \\
\ddot{0}\end{array}$ & 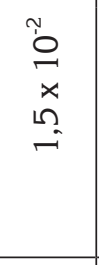 & $\begin{array}{l}-5 \\
0 \\
x \\
- \\
0\end{array}$ & 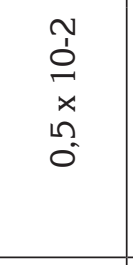 & $\begin{array}{l}\overrightarrow{0} \\
\times \\
x \\
0 \\
0\end{array}$ & $\begin{array}{l}\overline{1} \\
0 \\
x \\
a \\
0\end{array}$ & 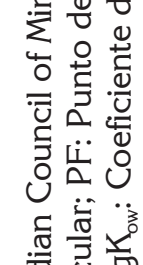 \\
\hline 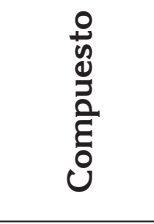 & $\begin{array}{l}\frac{0}{2} \\
\frac{0}{\pi} \\
\frac{0}{0} \\
\frac{0}{Z}\end{array}$ & 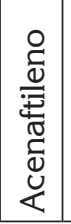 & 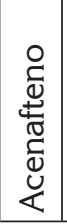 & $\left|\begin{array}{c}0 \\
\stackrel{0}{0} \\
\stackrel{\partial}{\partial} \\
\frac{\partial}{L}\end{array}\right|$ & 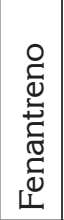 & 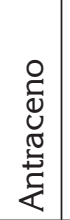 & $\begin{array}{c}0 \\
\stackrel{0}{0} \\
\stackrel{0}{0} \\
\frac{0}{0} \\
\frac{\partial}{L} \\
\end{array}$ & 导 & 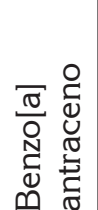 & 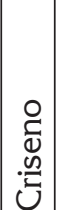 & 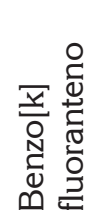 & 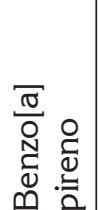 & 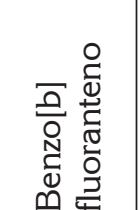 & 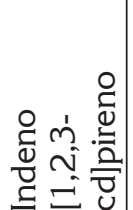 & 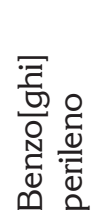 & 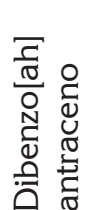 & 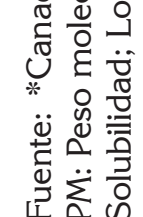 \\
\hline
\end{tabular}


La duración de los ensayos, se determinó teniendo en cuenta que para pruebas de bioacumulación en campo, la exposición abarca 30 días o más para evaluar concentraciones de contaminantes en tejidos; si los compuestos son debajo PM, este periodo puede ser inferior, pero para compuestos orgánicos de alto $\mathrm{PM}$, se puede prolongar la prueba a 60 o 90 días (APHA, AWWA, WEF, 2005).

La talla media de los organismos de ensayo fue de $4,88 \mathrm{~cm}$ de longitud total (LT), para A. tuberculosa y, de $4,48 \mathrm{~cm}$, para A. similis. La determinación de los niveles de HAP incorporados, se realizó con periodicidad de cinco días, tomando 10 organismos como muestra, extrayendo el tejido de los mismos y homogenizándolo, para realizar el análisis respectivo (a partir de $\pm 10 \mathrm{~g}$ de muestra).

La determinación de los HAP, se realizó por cromatografía de gases, acoplada a espectrometría de masas (CG/EM), siguiendo las metodologías descritas por Russell et al. (2002), utilizando un cromatógrafo de gases (Agilent Technologies $6890 \mathrm{~N}$ ), acoplado a un detector selectivo de masas (MSD 7683B Series); bajo el método de monitoreo de ion selectivo (SIM), empleando estándares de marca Supelco, de alta pureza analítica, para su determinación y su cuantificación.

Con el propósito de evaluar el desempeño analítico del método, se participó regularmente en pruebas interlaboratorio, con el Laboratorio Ambiental Marino (MEL) de la Agencia Internacional de Energía Atómica (IAEA), obteniendo resultados satisfactorios.

\section{RESULTADOS Y DISCUSIÓN}

Los parámetros fisicoquímicos presentaron valores entre los 23,30 y $25,70^{\circ} \mathrm{C}$, para la temperatura; de 7,91 a 8,24 , para el pH; entre 29,30 y 30,00 psu, para la salinidad y entre 3,25 y $7,80 \mathrm{mg} \mathrm{O}_{2} / \mathrm{L}$, para OD. Estos niveles son adecuados para la supervivencia de ambas especies, teniendo en cuenta que habitan en zonas fangosas en las que se presentan cambios bruscos en los diferentes parámetros fisicoquímicos, causados por los cambios mareales (Prahl et al. 1990).

Durante el periodo de aclimatación no se registró mortalidad $\mathrm{y}$, al término de este lapso, la concentración de los HAP totales presentó niveles de $143,50 \mathrm{ng} / \mathrm{g}$, en organismos de la especie A. tuberculosa (Tabla 2) y de $22,00 \mathrm{ng} / \mathrm{g}$, en $A$. similis (Tabla 3).

A lo largo de la prueba ninguna de las dos especies presentó incorporación de naftaleno ni de fluoranteno; esto obedece a que el naftaleno es el compuesto de menor PM entre los HAP y la tendencia en este grupo de compuestos es que se incrementa la volatilidad al disminuir el PM (CCME, 2010), lo que hace del naftaleno el compuesto con mayor volatilidad entre los HAP. Adicionalmente, la no incorporación de estos compuestos, también se puede relacionar con la baja concentración suministrada durante la prueba.

Este comportamiento es similar al registrado por algunos compuestos en mejillones expuestos a la fracción acomodada del crudo del Prestige, en el que se presentó una

Tabla 2. Concentración de HAP en Anadara tuberculosa durante la prueba de bioacumulación (ng/g - peso seco).

\begin{tabular}{|l|c|c|c|c|c|c|c|c|c|c|}
\hline \multicolumn{10}{|c|}{ Tiempo (días) } & \multicolumn{10}{|c|}{ CONCENTRACIÓN } \\
\cline { 2 - 11 } Compuesto & $\mathbf{0}$ & $\mathbf{5}$ & $\mathbf{1 0}$ & $\mathbf{1 5}$ & $\mathbf{2 0}$ & $\mathbf{2 5}$ & $\mathbf{3 0}$ & $\mathbf{3 5}$ & $\mathbf{4 0}$ & $\mathbf{4 5}$ \\
\hline Antraceno & 20,0 & 102,0 & 220,0 & 114,0 & 193,0 & 112,0 & 294,0 & 155,0 & 190,0 & 195,0 \\
\hline Pireno & 25,0 & 67,0 & 185,0 & 90,0 & 130,0 & 100,0 & 165,0 & 90,0 & 150,0 & 100,0 \\
\hline Benzo[k]fluoranteno & 1,6 & 57,0 & 110,0 & 114,0 & 163,0 & 122,0 & 172,0 & 226,0 & 100,0 & 130,0 \\
\hline Benzo[ghi]perileno & 1,7 & 40,0 & 140,0 & 120,0 & 118,0 & 110,0 & 216,0 & 155,0 & 110,0 & 130,0 \\
\hline Benzo[a]pireno & 4,7 & 32,0 & 85,0 & 69,0 & 130,0 & 75,0 & 153,0 & 142,0 & 75,0 & 950,0 \\
\hline Benzo[b]fluoranteno & 4,3 & 47,0 & 110,0 & 109,0 & 147,0 & 118,0 & 170,0 & 240,0 & 110,0 & 140,0 \\
\hline Benzo[a]antraceno & 1,7 & 54,0 & 100,0 & 87,0 & 160,0 & 100,0 & 148,0 & 175,0 & 98,0 & 120,0 \\
\hline Indeno[1,2,3-cd]pireno & 19,0 & 65,0 & 100,0 & 205,0 & 250,0 & 173,0 & 308,0 & 346,0 & 145,0 & 224,0 \\
\hline Dibenzo[ah]antraceno & 11,0 & 40,0 & 137,0 & 107,0 & 110,0 & 96,0 & 220,0 & 168,0 & 113,0 & 140,0 \\
\hline Fluoranteno & $<$ L.D & $<$ L.D & $<$ L.D & $<$ L.D & $<$ L.D & $<$ L.D & $<$ L.D & $<$ L.D & $<$ L.D & $<$ L.D \\
\hline Criseno & 0,5 & 102,0 & 140,0 & 161,0 & 270,0 & 173,0 & 250,0 & 332,0 & 150,0 & 210,0 \\
\hline Naftaleno & $<$ L.D & $<$ L.D & $<$ L.D & $<$ L.D & $<$ L.D & $<$ L.D & $<$ L.D & $<$ L.D & $<$ L.D & $<$ L.D \\
\hline Acenaftileno & 15,0 & 313,0 & 200,0 & 564,0 & 590,0 & 364,0 & 370,0 & 540,0 & 530,0 & 610,0 \\
\hline Acenafteno & 13,0 & 757,0 & 347,0 & 1236,0 & 1210,0 & 838,0 & 790,0 & 1156,0 & 1070,0 & 1290,0 \\
\hline Fluoreno & 14,0 & 440,0 & 480,0 & 575,0 & 630,0 & 506,0 & 704,0 & 637,0 & 610,0 & 730,0 \\
\hline Fenantreno & 12,0 & 320,0 & 510,0 & 360,0 & 410,0 & 350,0 & 600,0 & 440,0 & 420,0 & 520,0 \\
\hline Sumatoria HAP & 143,5 & 2436,0 & 2864,0 & 3911,0 & 4511,0 & 3237,0 & 4560,0 & 4802,0 & 3871,0 & 5489,0 \\
\hline
\end{tabular}

< L.D: Menor al límite de detección. 
Tabla 3. Concentración de HAP en Anadara similis durante la prueba de bioacumulación (ng/g - peso seco).

\begin{tabular}{|c|c|c|c|c|c|c|c|}
\hline \multirow{2}{*}{ Compuesto } & \multicolumn{7}{|c|}{ CONCENTRACIÓN } \\
\hline & 0 & 5 & 10 & 15 & 20 & 25 & 30 \\
\hline Antraceno & 0,7 & 114,0 & 114,0 & 145,0 & 280,0 & 375,0 & 194,0 \\
\hline Pireno & 8,7 & 50,0 & 180,0 & 204,0 & 166,0 & 340,0 & 220,0 \\
\hline Benzo[k]fluoranteno & $<$ L.D & 130,0 & 93,0 & 122,0 & 40,0 & 120,0 & 166,0 \\
\hline Benzo[ghi]perileno & $<$ L.D & 84,0 & 55,0 & 64,0 & 90,0 & 80,0 & 100,0 \\
\hline Benzo[a]pireno & $<$ L.D & 68,0 & 46,0 & 56,0 & 27,0 & 94,0 & 90,0 \\
\hline Benzo[b]fluoranteno & $<$ L.D & 95,0 & 96,0 & 120,0 & 56,0 & 140,0 & 193,0 \\
\hline Benzo[a]antraceno & $<$ L.D & 84,0 & 104,0 & 120,0 & 80,0 & 214,0 & 206,0 \\
\hline Indeno[1,2,3-cd]pireno & $<$ L.D & 107,0 & 83,0 & 115,0 & 36,0 & 136,0 & 147,0 \\
\hline Dibenzo[ah]antraceno & $<$ L.D & 83,0 & 60,0 & 77,0 & 24,0 & 96,0 & 96,0 \\
\hline Fluoranteno & $<$ L.D & $<$ L.D & $<$ L.D & $<$ L.D & $<$ L.D & $<$ L.D & $<$ L.D \\
\hline Criseno & 5,0 & 160,0 & 222,0 & 315,0 & 176,0 & 410,0 & 460,0 \\
\hline Naftaleno & $<$ L.D & $<$ L.D & $<$ L.D & $<$ L.D & $<$ L.D & $<$ L.D & $<$ L.D \\
\hline Acenaftileno & 3,4 & 135,0 & 40,0 & 814,0 & 1040,0 & 1100,0 & 645,0 \\
\hline Acenafteno & $<$ L.D & 504,0 & 153,0 & 1930,0 & 2247,0 & 2320,0 & 1550,0 \\
\hline Fluoreno & 4,4 & 536,0 & 425,0 & 880,0 & 1144,0 & 1150,0 & 840,0 \\
\hline Fenantreno & $<$ L.D & 600,0 & 678,0 & 702,0 & 865,0 & 930,0 & 670,0 \\
\hline Sumatoria HAP & 22,0 & 2750,0 & 2380,0 & 5664,0 & 6270,0 & 7505,0 & 5580,0 \\
\hline
\end{tabular}

$<$ L.D: Menor al límite de detección.

baja bioacumulación de HAP, que podría estar relacionada con la presencia de compuestos de bajo PM, los que pueden ser rápidamente liberados a la atmósfera y, por lo tanto, no son disponibles para los organismos acuáticos (Solé et al. 2007)

Los compuestos restantes fueron incorporados por los organismos en mayor proporción para aquellos de alto $\mathrm{PM}$, teniendo en cuenta los niveles determinados frente a las concentraciones suministradas. A este comportamiento, se suma una baja variabilidad a lo largo del periodo de exposición; mientras que los compuestos de menor PM presentaron un comportamiento inverso, situación derivada de la mayor disponibilidad y menor persistencia que les caracteriza.

Los máximos niveles de HAP en los organismos, se registraron luego de 25 días de exposición, en $A$. similis, alcanzando una concentración de 75,05 x 10² ng/g (Tabla 3), frente a 45,60 x $10^{2} \mathrm{ng} / \mathrm{g}$, para el mismo periodo de tiempo, en $A$. tuberculosa, cuya máxima concentración se registró luego de 35 días de prueba, con 48,00x 10²ng/g (Tabla 2), lo que indica que la velocidad y la capacidad de incorporación de estos compuestos, por parte de la primera especie, es mucho mayor.
En cuanto a los niveles de HAP incorporados por los organismos, estos fueron mayores para el acenafteno y, fluoreno, en ambas especies, siguiéndole en concentración el fenantreno y el acenaftileno, en $A$. similis $\mathrm{y}$, ambos compuestos, de manera invertida, en A. tuberculosa, como se puede observar en las figuras 2 y 3 . Los compuestos de bajo PM presentaron oscilaciones importantes durante las pruebas y una mayor incorporación con respecto a los de alto PM.

Este comportamiento es similar al presentado por el mejillón Mytilus galloprovincialis, al exponerse bajo condiciones de laboratorio, a concentraciones de HAP, pues en esta especie se registró una mayor acumulación de compuestos de bajo PM (Baussant et al. 2001a,b; Neff, 2002; Meador, 2003). Así mismo, se encontró que la depuración de los compuestos de menor PM y mayor solubilidad en agua, como el naftaleno, fue mucho más rápida (Baussant et al. 2001a,b).

El benzo[a]pireno registró la menor concentración acumulada por ambas especies, mientras que los compuestos restantes no presentaron un patrón o relación en cuanto a acumulación comparable entre ellas; sin embargo, se observó un comportamiento bastante similar entre los compuestos de alto PM, en ambas especies. 


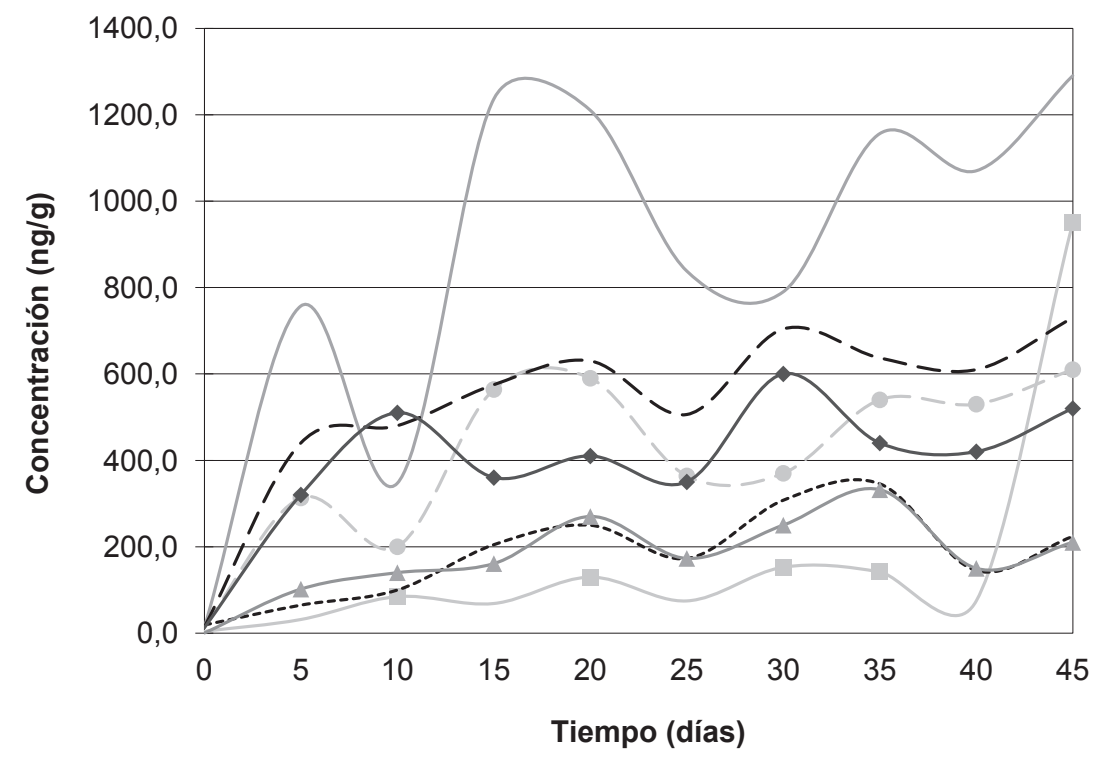

\author{
Benzo[a]pireno \\ Indeno[1,2,3-cd] $\mathrm{F}$ \\ Criseno \\ Acenaftileno \\ Acenafteno \\ Fluoreno \\ Fenantreno
}

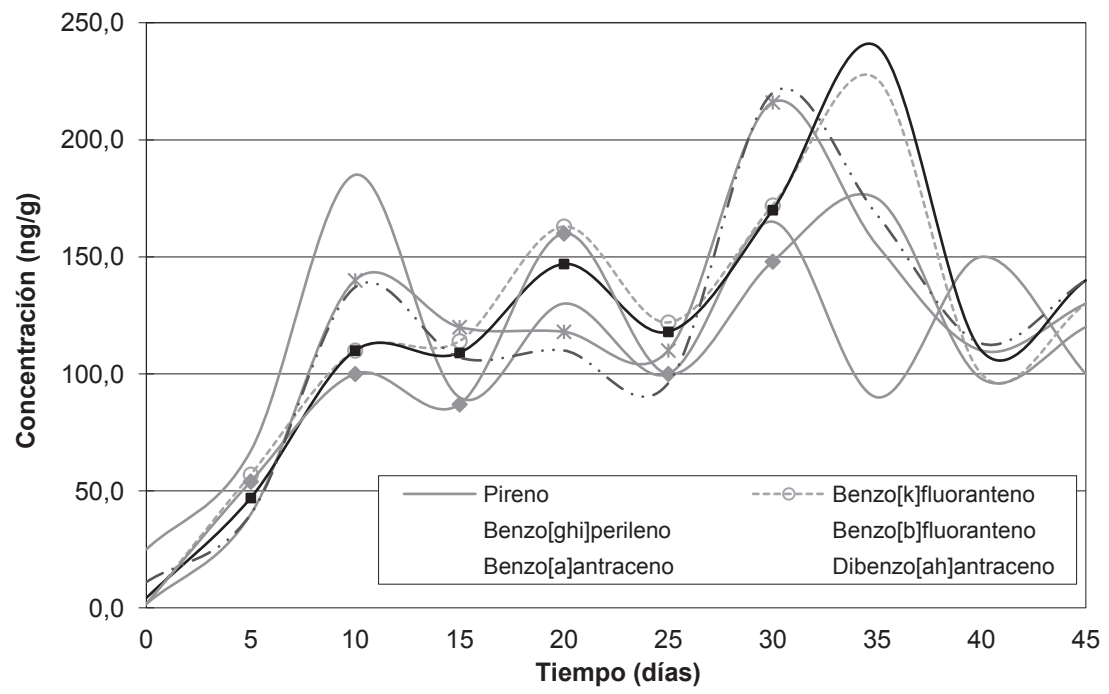

Figura 2. Comportamiento de HAP en Anadara tuberculosa durante la prueba de bioacumulación.

El reglamento de la Comisión Europea CE (2011) establece que el contenido máximo de HAP en moluscos bivalvos (frescos, refrigerados o congelados) se ha estimado en 5,0ng/g, para el benzo[a]pireno; sin embargo, como es posible que el mismo no se encuentre acumulado por los organismos, se ha planteado una concentración de $30 \mathrm{ng} / \mathrm{g}$ para la suma de benzo[a]pireno benzo[a]antraceno, benzo[b] fluoranteno y criseno, con el fin de emplear estos cuatro compuestos como un marcador o indicador de toxicidad, para los HAP.

Teniendo en cuenta este reglamento, las concentraciones de HAP determinadas para los organismos de ensayo (Tablas 2 y 3), evidencian que los mismos solamente se encontraban aptos para el consumo humano antes de dar inicio a las pruebas (tras varios días, bajo condiciones de aclimatación), pues luego de ser expuestos a la mezcla de HAP, tanto el nivel de benzo[a]pireno como el de la sumatoria de compuestos relacionados como indicadores, superaron el contenido máximo estipulado.

Las concentraciones de HAP en los organismos presentaron variaciones a lo largo de las pruebas, comportamiento usual ante la exposición a contaminantes en el medio, ya que los organismos no solo incorporan los contaminantes, sino que, a la vez, pueden realizar excreción de los mismos, 

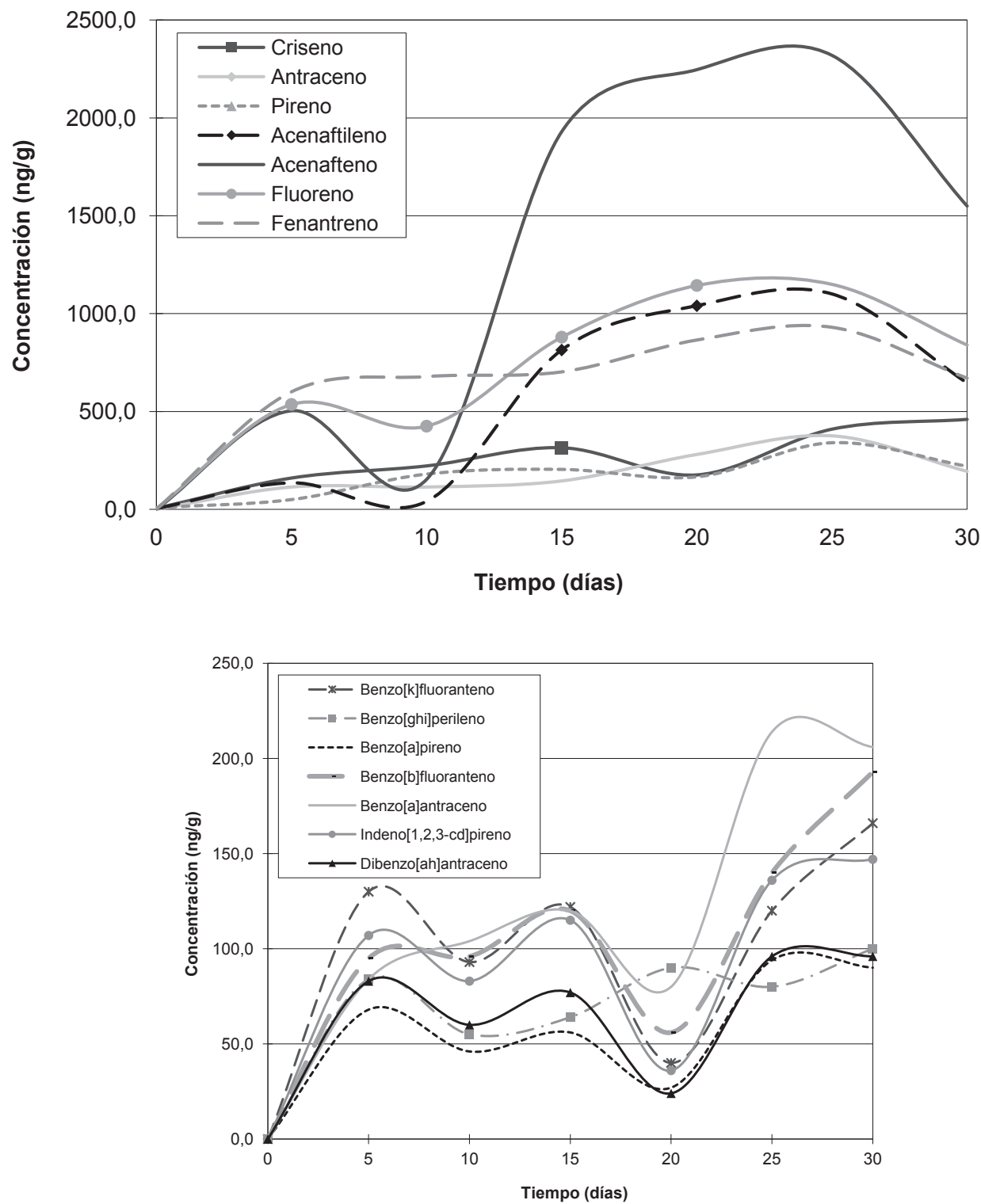

Figura 3. Comportamiento de HAP en Anadara similis durante la prueba de bioacumulación.

aumentando y disminuyendo las concentraciones en sus tejidos, aún en presencia de los compuestos en el medio (Calero \& Zambrano, 1997; CSIC, 2003; Prada-Ríos \& Zambrano-Ortiz, 2006).

Estos procesos de incorporación y de depuración han sido reportados en organismos presentes en el ambiente natural, tras evaluaciones en zonas afectadas por el ingreso de petróleo al medio marino, presentando, inicialmente, un proceso rápido de incorporación y, luego, los compuestos son excretados y disminuyen o se eliminan totalmente, cuando la contaminación en el medio desciende o desaparece (CSIC, 2003).
Lo que define la mayor o menor capacidad de bioacumulación de un compuesto es el tamaño de la molécula, que al aumentar el PM genera un incremento en la persistencia, en la toxicidad y en el carácter lipofílico, siendo los organismos acuáticos altamente afectados por estas características de los HAP (Antón \& Lizaso, 2003).

Ambas especies registraron mortalidad a lo largo de la prueba, alcanzando un $43,50 \%$, en $A$. tuberculosa (entre los días dos y 29) y, un $48,50 \%$, en $A$. similis (del día dos al 21 , con un alto porcentaje entre los días dos y 12). En los recipientes de control, $A$. tuberculosa alcanzó una mortalidad del 33,33\% (entre los días 13 y 33) y, de un 53,33\%, en 
A. similis (entre los días siete y 18), en el control. En los recipientes a los que se adicionó acetona, se registró una mortalidad correspondiente al $56,66 \%$, para A. tuberculosa (en los días 16 y 22) y, del 66,66\%, A. similis (entre los días cinco y 13$)$.

Esta tasa de mortalidad, se puede relacionar con una alta sensibilidad de los organismos expuestos, pues no solo se presentó en los recipientes que contenían la mezcla de HAP, sino también en los dos tipos de recipientes de control. Sin embargo, debido a que durante el periodo de aclimatación (10 días) la sobrevivencia fue total, es claro que los organismos se encontraban en óptimas condiciones al inicio del ensayo.

Por otra parte, el que se registrara mortalidad en los organismos de ambos tipos de control (agua de disolución y agua-acetona) descarta la posibilidad que la misma se asociara exclusivamente con la exposición al solvente o a la mezcla suministrada. Esta mortalidad, evidencia la presencia de un factor adicional a la mezcla de HAP suministrada, que ejerció una influencia negativa sobre los organismos, probablemente, asociado con el periodo previo de aclimatación, pues pruebas de bioacumulación en las que A. tuberculosa se expuso a concentraciones de HAP, de manera individual o en mezcla bajo condiciones similares a la del presente ensayo, y en las que este periodo no superó los cinco días, no se registró mortalidad en los recipientes de control ni en aquellos en que se expusieron los organismos a la solución de prueba (Calero \& Zambrano, 1997; PradaRíos \& Zambrano-Ortiz, 2006).

El periodo de aclimatación fue mayor al empleado en bioensayos previos, con el fin que los organismos depuraran los niveles de HAP que pudieran haber incorporado del medio antes de su captura.

El cese total de la mortalidad transcurrido en un determinado periodo de tiempo evidencia una respuesta de adaptación por parte de los organismos, tras una mayor sensibilidad inicial que, sin duda, fue acentuada por la presencia de la mezcla tóxica en el medio. Esta "adaptabilidad", se puede comparar con las respuestas presentadas por los organismos en el medio natural, después del ingreso de compuestos tóxicos que, en principio, genera una alta mortalidad, la cual, disminuye paulatinamente.

Los altos niveles de HAP incorporados por los organismos, no solo ratifican la importancia de las especies de bivalvos en los estudios de evaluación de contaminantes, sino que, a la vez, evidencian el alto riesgo que representa para la salud de los consumidores de estos productos, situación que se potencializa cuando los mismos provienen de áreas contaminadas.
La alta capacidad de bioacumulación, especialmente, presentada por parte de A. similis frente a A. tuberculosa, evidencia un mayor potencial de la misma como especie biomonitora; así mismo, el mayor porcentaje de mortalidad registrado por esta especie puede estar influenciado por los altos niveles de contaminantes incorporados en cortos periodos de tiempo y, a la vez, sugiere una mayor sensibilidad de la especie ante condiciones adversas.

Estas características no solo ratifican la importancia y el potencial de ambas especies como organismos biomonitores, sino que resultan muy importantes, si se tiene en cuenta que se ha determinado que especies de bivalvos, como Mya arenaria, tras acumular concentraciones elevadas de HAP, necesitan varias semanas en tanques de depuración para reducirlas a niveles insignificantes (Lee et al. 2010), lo que, sin duda, evidencia la necesidad de realizar seguimientos a las concentraciones de estos compuestos en las especies estudiadas, con el fin de evaluar su calidad, teniendo en cuenta la gran demanda para su consumo por parte de las poblaciones, ubicadas en el litoral Pacífico colombiano y en otros países de la región.

Agradecimientos: Los autores agradecen a la Dirección del Centro de Investigaciones Oceanográficas e Hidrográficas del Pacífico - CCCP de la Dirección General Marítima DIMAR, por la financiación para el desarrollo del presente estudio y al personal del laboratorio de química por su valiosa colaboración en los análisis de las muestras. Conflicto de intereses: El manuscrito fue preparado y revisado con la participación de todos los autores, quienes declaramos que no existe ningún conflicto de intereses que ponga en riesgo la validez de los resultados presentados. Financiación: Este estudio fue financiado por el Centro de Investigaciones Oceanográficas e Hidrográficas del Pacífico - CCCP.

\section{BIBLIOGRAFÍA}

1. ACHTEN, C.; HOFMANN, T. 2009. Native polycyclic aromatic hydrocarbons (PAH) in coals - a hardly recognized source of environmental contamination. Sci. Total Environ. 407:2461-2473.

2. ANTÓN, A.; LIZASO, J. 2003. Hidrocarburos aromáticos policíclicos. Fundación Ibérica para la Seguridad Alimentaria. Madrid (España). 225 p.

3. APHA, AWWA, WEF. 2005. Standard Methods for the Examination of Water and Wastewater21st ed. American Public Health Association. Washington, DC. (Estados Unidos). Part 8000. 8, p.94-100. 
4. ARDILA, N.; NAVAS, G.R.; REYES, J. (Eds.). 2002. Libro rojo de invertebrados marinos de Colombia. INVEMAR. Ministerio de Medio Ambiente. La serie Libros rojos de especies amenazadas de Colombia. Bogotá (Colombia). p.96-98.

5. ATSDR. 2011. 2007 CERCLA Priority List of Hazardous Substances. Atlanta, GA, (Estados Unidos). Disponible desde Internet en: www.atsdr.cdc.gov/cercla/07list. html (con acceso 26/04/2011).

6. BAUSSANT, T.; SANNI, S.; JONSSON, G.; SKADSHEIM, A.; BORSETH, J.F. 2001a. Bioaccumulation of polycyclic aromatic compounds: 1. Bioconcentration in two marine species and in semipermeable membrane devices during chronic exposure to dispersed crude oil. Environ. Toxicol. Chem. 20 (6):1175-1184.

7. BAUSSANT, T.; SANNI, S.; SKADSHEIM, A.; JONSSON, G.; BORSETH, J.F.; GAUDEBERT, B. 2001b. Bioaccumulation of polycyclic aromatic compounds: 2. Modeling bioaccumulation in marine organisms chronically exposed to dispersed oil. Environ. Toxicol. Chem. 20(6):1185-1195.

8. BELIAEFF, B.; O'CONNOR, T.P.; DASKALAKIS, D.K.; SMITH, P.J. 1997. U.S. Mussel Watch data from 1986-1994: Temporal Trend detection at large spatial scales. Environ. Sci. Technol. 3:1411-1415.

9. BELLAS, J.; SACO-ÁLVAREZ, L.; NIETO, O.; BEIRAS, R. 2008. Ecotoxicological evaluation of polycyclic aromatic hydrocarbons using marine invertebrate embryo-larval bioassays. Mar. Pollut. Bull. 57:493502.

10. CALERO, L.; ZAMBRANO, M. 1997. Bioacumulación de hidrocarburos aromáticos del petróleo en un molusco bivalvo Anadara tuberculosa. Bol. Cient. Cccp. (Colombia). 6:131-137.

11. CASANOVA, R. 1995. Estudio de la contaminación marina por compuestos organoclorados en el Pacífico colombiano. Bol. Cient. Cccp. (Colombia). 5:146160.

12. CCME, Canadian Council of Ministers of the Environment. 2010. Canadian Soil Quality Guidelines for Carcinogenic and Other Polycyclic Aromatic Hydrocarbons (Environm. and Human Health Effects). Sci. Criteria Document (revised). Quebec (Canada). 216p.
13. CE, Comisión Europea. 2011. REGLAMENTO (UE) No. 835/2011 de la Comisión de 19 de agosto de 2011. Contenido máximo de hidrocarburos aromáticos policíclicos en los productos alimenticios. Diario oficial de la Unión Europea. Bruselas (Bélgica). L 215/4-8. Disponible desde Internet en: http://eur-lex. europa.eu/LexUriServ/LexUriServ.do?uri=OJ:L:2011: 215:0004:0008:ES:PDF (con acceso 22/05/2012).

14. CHEĆ, E.; PODGÓRSKA, B.; WĘGRZYN, G. 2008. Comparison of the use of mussels and semipermeable membrane devices for monitoring and assessment of accumulation of mutagenic pollutants in marine environment in combination with a novel microbiological mutagenicity assay. Environ. Monit. Assess. 140:83-90.

15. CSIC. 2003. Impacto de un vertido de petróleo sobre los organismos marinos. Algunas lecciones del vertido del Aegean sea. Informe técnico No. 15. (España). Disponible desde Internet en: http://csicprestige.iim. csic.es/informes/info15.pdf (con acceso 03/04/2011).

16. DE LA CRUZ, E. 1994. Stable pollutants in the bivalve Anadara tuberculosa, from the Nicoya Gulf, Costa Rica. En: Otchere, F.A. 2005. Organochlorines (PCBs and pesticides) in the bivalves Anadara (Senilis) senilis, Crassostrea tulipa and Perna perna from the lagoons of Ghana. Sci. Total. Environ. 348:102-114.

17. Diario Oficial de la Unión Europea. 2011. REGLAMENTO (UE) No. 835/2011 DE LA COMISIÓN de 19 de agosto de 2011. Contenido máximo de hidrocarburos aromáticos policíclicos en los productos alimenticios. Bruselas. Bélgica. 5p.

18. EISLER, R. 2000. Polycyclic aromatic hydrocarbons. In: Eisler, R. (ed.) Handbook of chemical risk assessment. Volume II. Boca Raton, FL. Lewis Publishers (Estados Unidos). p.1343-1411.

19. HERRERO, L.; PALACIOS, A.; HUN, L.; VEGA, F. 1999. Ausencia de detección de enterovirus en bivalvos Anadara tuberculosa (Bivalvia: Arcidae) por contaminación química en el Pacífico de Costa Rica. Rev. Biol. Trop. (Costa Rica). 47:419-427.

20. IARC. 1983. Polynuclear Aromatic Compounds: Part 1, Chemical, environmental and experimental data. IARC Monographs on the Evaluation of Carcinogenic Risks to Humans. World Health Organization. 32:95431. (Francia). Last updated: 17 April 1998. 
21. INCODER. 2007. Sistema de información sectorial pesquero. En: Lovatelli, A.; Farias, A.; Uriarte, I. (eds). Estado actual del cultivo y manejo de moluscos bivalvos y su proyección futura: factores que afectan su sustentabilidad en América Latina. Taller Técnico Regional de la FAO. 20-24 de agosto de 2007, Puerto Montt, Chile. FAO Actas de Pesca y Acuicultura. No. 12. Roma, FAO. p.115-128.

22. LAUENSTEIN, G.G. 1995. Comparison of organic contaminants founding mussels and oysters from a current Mussel Watch Project with those from archived mollusk samples of the 1970s. Mar. Pollut. Bull. 30:826-833.

23. LEE, R.; LOVATELLI, A.; ABABOUCH, L. 2010. Depuración de bivalvos: Aspectos fundamentales y prácticos. FAO. Documento Técnico de Pesca. No. 511. Roma. (Italia). 153p.

24. MARRUGO, A.J. 1993. Estudio de la contaminación marina por hidrocarburos en el Pacífico Colombiano - Fase III. Bol. Cient. Cccp. (Colombia). 4:47-60.

25. MEADOR, J.P. 2003. Bioaccumulation of pahs in marine invertebrates. in: Douben, P.E.T. (ed.) PAHs: An ecotoxicological perspective. John Wiley \& Sons Ltd. Londres (Reino Unido). p.147-171.

26. NEFF, J.M. 2002. Bioaccumulation in marine organisms. Effect of contaminants from oil well produced water. Elsevier Science Publishers. Oxford. (Reino Unido). 452p.

27. NESTO, N.; CASSIN, D.; DA ROS, L. 2010. Is the polychaete, Perinereis rullieri (Pilato 1974), a reliable indicator of PCB and PAH contaminants in coastal sediments? Ecotox. Environ. Safe. 73:143-151.

28. OKAY, O.S.; KARACIK, B. 2008. Bioconcentration and phototoxicity of selected PAHs to marine mussel Mytilus galloprovincialis. J. Environ. Sci. Health. 43(11):1234-1242.

29. PAGE, D.S.; BOEHM, P.D.; BROWN, J.S.; NEFF, J.M.; BURNS, W.A.; BENCE, A.E. 2005. Mussels document loss of bioavailable polycyclic aromatic hydrocarbons and the return to baseline conditions for oiled shorelines in Prince William Sound, Alaska. Mar. Environ. Res. 60:422-436.

30. PELLETIER, E.; SARGAIN, P.; PAYET, J.; DEMERS, S. 2006. Ecotoxicological effects of combined UVB and organic contaminants in coastal waters: a review. Photochem. Photobiol. 82(4):981-993.

31. POTIN, O.; RAFIN, C.; VEIGNIE, E. 2004. Bioremediation of aged polycyclic aromatic hydrocarbons (PAHs)contaminated soil by filamentous fungi isolated from the soil. Int. Biodeter. Biodegr. 54(1):45-52.

32. PRADA-RÍOS, J.E.; ZAMBRANO-ORTIZ, M.M. 2006. Toxicidad aguda y bioacumulación de dos hidrocarburos aromáticos policíclicos (naftaleno y fluoranteno) en Anadara tuberculosa. Bol. Cient. Cccp. (Colombia). 13:53-64.

33. PRAHL, H. von; CANTERA, J.R.; CONTRERAS, R. 1990. Manglares y hombres del Pacífico colombiano. Fondo FEN. Bogotá (Colombia). 193p.

34. REY-SALGUEIRO, L.; MARTÍNEZ-CARBALLO, E.; GARCÍA-FALCÓN, M.; SIMAL-GÁNDARA, J. 2009. Survey of polycyclic aromatic hydrocarbons in canned bivalves and investigation of their potential sources. Food. Res. Int. 42:983-988.

35. RUSSELL, M.L.; GOTH-GOLDSTEIN, R.; APTE, M.G.; FISK, W.J. 2002. Analytical Method for the determination of Individual ( $\mathrm{N}$-alkanes and Isoprenoids) and Total Petroleum Hydrocarbons (TPH), Polycyclic Aromatic Hydrocarbons (PAHs) and Biomarker Triterpanes and Steranes in Oil and Oil-spill-related. Environmental Samples (Water, Particulates and Soils/Sediment): Berkeley (CA): LawrenceBerkeley National Laboratory, Environmental Energy Technologies Division; 2002 Jan. Report No.: LBNL49574. Contract No.: DEAC0376SF00098. Sponsored by the Department of Energy. Ottawa (Canada), ON, K1A OH3.

36. SERICANO, J.L.; WADE, T.L.; JACKSON, T.J.; BROOKS, J.M.; TRIPP, B.W.; FARRINGTON, J.W.; MEE, L.D.; READMAN, J.W.; VILLENEUVE, J.P.; GOLDBERGS, E.D. 1995. Trace organic contamination in the Americas: an overview of the US National Status \& Trends and the International "Mussel Watch" Programmes. Mar. Pollut. Bull. 31(4-12):214-225.

37. SOLÉ, M.; BUET, A.; ORTIZ, L.; MAYNOU, F.; BAYONA, J.J.; ALBAIGÉS, J. 2007. Bioaccumulation and biochemical responses in mussels exposed to the water-accommodated fraction of the Prestige fuel oil. Sci. Mar. (España). 71(2):373-382.

38. SORIANO-SANZ, J.A.; FRANCO-HERNÁNDEZ, A.; VIÑAS-DIÉGUEZ, L.; CAMBEIRO-CAMBEIRO, 
B.; GONZÁLEZ-FERNÁNDEZ， J.J. 2006. Datos preliminares de hidrocarburos aromáticos policíclicos en mejillón silvestre de la costa cantábrica (España) después del vertido del Prestige. Cienc. Mar. (España). 32(2B):457-463.

39. VELÁSQUEZ LÓPEZ, O.; CORTES, L.M. 1997. Estudio y evaluación de metales traza $(\mathrm{Pb}, \mathrm{Cr}, \mathrm{Cd}$ y $\mathrm{Hg})$ en aguas, sedimentos y organismos marinos de la bahía de Buenaventura. Bol. Cient. Cccp. (Colombia). 6:5761.

40. ZEDECK, M.S. 1980. Polycyclic aromatic hydrocarbons: a review. J Environ. Pathol. Tox. 3:537-567.

Recibido: Octubre 11 de 2011

Aceptado: Agosto 17 de 2012 